\title{
Quadratic Polynomial Form of Electric Arc Furnace Equation
}

\author{
Dariusz Grabowski \\ Faculty of Electrical Engineering \\ Silesian University of Technology \\ Gliwice, Poland \\ dariusz.grabowski@polsl.pl
}

\begin{abstract}
A closed form solution to a differential equation used as the deterministic AC electric arc model exists only for some arc parameter values. The paper shows that periodic solutions for the other parameter values can be found by combining the harmonic balance method (HBM) and a continuation method. In order to avoid problems occurring when a large number of harmonics is required or the system contains strong nonlinearities, a transformation of the original equation has been proposed. Nonlinearities present in the equation have been transformed into purely polynomial quadratic terms. It facilitates application of the classical HBM and allows to follow periodic solutions of the arc equation when its parameters are varied. It also enables better understanding of the phenomena described by the equation and makes easier the extension of the arc model in order to cover the time-varying character of the arc waveforms. Theoretical considerations have been illustrated by a simulation experiment.
\end{abstract}

Keywords-arc furnace; nonlinear elements; modeling; HBM

\section{INTRODUCTION}

Understanding properties of solutions to differential equations describing physical phenomena is fundamental for modern engineering. A differential equation describing the AC electric arc is just one of the examples. Electric arc furnaces are commonly used for melting metals in steel industry. Unfortunately, nonlinear characteristic of the arc furnace and its stochastic behavior are the cause of voltage flicker and waveform distortions in power systems [1], [2].

The stochastic nature of processes which take place in the furnace makes the development of a realistic arc model a challenging task. However, a reliable model is required to estimate the degradation of the power quality caused by the arc furnace and to take some actions which enable to overcome the mentioned above negative effects.

During melting the arc extinguishes and starts again in a random way. In spite of the arc stochastic nature, its analysis usually starts using deterministic characteristic and the timevarying nature is taken into account in the second step. It is made with the aid of chaotic, stochastic or modulated components which are added to the deterministic solution [2], [3], [4].
This paper is a continuation of works [5] and [6] in which a closed form solution to one of the most popular deterministic nonlinear differential equations used for the arc modeling [7] has been developed for some arc parameters. It seems that a closed form solution for the other arc parameters does not exists [8]. However, in this case periodic solutions can be found by combining the harmonic balance method (HBM) and a continuation method [9]. A transformation of the nonlinearities present in the equation into purely polynomial quadratic terms has been proposed in the paper. It makes the determination of periodic solutions easier.

The final task consists in considering some parameters of the arc as stochastic variables to obtain a realistic arc model. This task will be for sure easier to accomplish if the closed form solutions or periodic solutions are analyzed instead of using the numerical approach.

\section{ARC FURNACE MODEL}

Deterministic and stochastic components can be observed in voltage-current (V-I) arc characteristics. The share of both components depends on the phase of the melting process [2].

Modeling of the deterministic V-I characteristic can be made with the help of nonlinear differential equation [2], [3], [7], piece-wise linear approximation [4], mixed approximation using exponential and linear functions [4], approximation using shifted and amplified step function [10]. The paper follows the first mentioned above approach. The following nonlinear differential equation describing a single-phase electric arc can be derived from the power balance equation [7]:

$$
k_{1} r^{n}(t)+k_{2} r(t) \frac{d r(t)}{d t}=\frac{k_{3}}{r^{m+2}(t)} i^{2}(t),
$$

where:

$$
\begin{aligned}
& r(t) \text { - the arc radius, } \\
& i(t) \text { - the arc current, } \\
& k_{j} \text { - the proportionality constants, } j=1,2,3, \\
& n, m \text { - the arc parameters, } n=0,1,2, m=0,1,2 .
\end{aligned}
$$


The current waveform $i(t)$ is treated as an input data when analyzing the arc phenomenon. Equation (1) can be also used to get the characteristic of a three-phase electric arc [2].

The general arc equation (1) is a first-order nonlinear differential equation. Unfortunately, there are no general analytical methods which allow to solve any nonlinear differential equation, even of the first-order. In that case the general arc equation (1) is nor separable neither exact, so there is no simple way to find its solution in a closed form [8].

\section{PERIODIC SOLUTION TO THE ARC EQUATION}

Using a substitution given in [6] allows to convert (1) into the following first-order differential equation:

$$
\frac{d y(t)}{d t}=\alpha y^{k}(t)+f(t)
$$

where:

$$
f(t)=\frac{(m+4) k_{3}}{k_{2}} i^{2}(t), \alpha=-\frac{(m+4) k_{1}}{k_{2}}, k=\frac{n+m+2}{m+4}
$$

For $n=2$ and $m=0,1$ or 2 the coefficient $k$ is equal 1 and the linear first-order differential equation is obtained. A closed form solution to such equation is well known [8]. On the base of this solution the arc radius $r(t)$ and subsequently the arc voltage $v(t)$ can be determined [7] without need for numerical solution to the nonlinear differential equation (1).

For $n=0$ and $m=0,1,2$ the coefficient $k$ is equal to $1 / 2$, $3 / 5$ and $2 / 3$. For $n=1$ and $m=0,1$ or 2 the coefficient $k$ is equal to $3 / 4,4 / 5$ and $5 / 6$. In both cases, the HBM, which consists in decomposing the $y(t)$ into a truncated Fourier series and substitution of the series into the right hand side of (2), may be applied. The resultant system of algebraic equations enables to determine the amplitudes of all the harmonics and thus the periodic solution $y(t)$. The application of the HBM becomes straightforward if the nonlinear equation is transformed to a new quadratic form [9]:

$$
m(\dot{\mathbf{z}})=c(t)+l(\mathbf{z})+q(\mathbf{z}, \mathbf{z}),
$$

where:

$$
\begin{aligned}
& m(\cdot), l(\cdot) \text { - linear vector operators, } \\
& q(\cdot, \cdot) \text { - a quadratic vector operator, } \\
& c(t) \text { - a forcing and constant term vector. }
\end{aligned}
$$

The system of equations (4) can contain both differential (the dot stands for the derivative with respect to time) and algebraic equations. The vector $\mathbf{z}$ contains the original unknown variable $y$ and some additional variables introduced to obtain the quadratic form.

The quadratic polynomial form of (2) for all mentioned above values of the coefficient $k$ has been given in the paper. For example, if $k=5 / 6$, then:

$$
\begin{aligned}
\dot{y} & =f(t)+\alpha w \\
0 & =0+v-y^{2} \\
0 & =0+s-v^{2} \\
0 & =0+x-w^{2} \\
0 & =0+p-x^{2} \\
\underbrace{0}_{m(\mathbf{z})} & =\underbrace{0}_{c(t)}+\underbrace{0}_{l(\alpha, \mathbf{z})}+\underbrace{y s-x p}_{q(\mathbf{z}, \mathbf{z})}
\end{aligned}
$$

The results of application of the HBM to (4) obtained for the nonlinear differential equations describing the arc phenomena have been presented in the paper. The theoretical results have been illustrated by examples.

\section{CONCLUSIONS}

The nonlinear differential equation used to model the electric arc furnace has been transformed to the quadratic polynomial form. Since the nonlinearities are quadratic, the HBM can be easily applied to the transformed equation, even with a large number of harmonics. In order to follow periodic solutions of the arc equation when its parameters are varied a continuation method can be applied in the next step. The computational results obtained so far agree with existing numerical solutions and measurements.

\section{REFERENCES}

[1] G. Manchur and C.C. Erven, "Development of a model for predicting flicker from arc furnaces," IEEE Trans. Power Delivery, vol. 7, pp. 416426, Jan. 1992.

[2] A. A. Gomez, J.J.M. Durango and A. E. Mejia, "Electric arc furnace modeling for power quality analysis," Proc. of the IEEE ANDESCON Conf., 14-17 Sept., Bogota, pp. 1-6, 2010.

[3] O. Ozgun and A. Abur, "Development of an arc furnace model for power quality studies," Proc. IEEE -PES Summer Meeting 1999, vol. 1, pp. 507-511, 1999.

[4] M.A. Golkar and S. Meschi, "MATLAB modeling of arc furnace for flicker study," Proc. of the IEEE Int. Conf. on Industrial Technology, 21-24 April, Chengdu, pp. 1-6, 2008.

[5] D. Grabowski and J. Walczak, "Analysis of deterministic model of electric arc furnace," Proc. of 10th International Conference on Environment and Electrical Engineering EEEIC, Rome, Italy, pp. 1-4, May 2011.

[6] D. Grabowski and J. Walczak, "Deterministic model of electric arc furnace - a closed form solution," The International Journal for Computation and Mathematics in Electrical and Electronic Engineering COMPEL, vol. 32, no. 4, in press.

[7] E. Acha, A. Semlyen and N. Rajakovic, "A harmonic domain computational package for nonlinear problems and its applications to electric arcs", IEEE Trans. on Power Delivery, vol. 5, pp. 1390-1397, Jul. 1990.

[8] D. Zwillinger, Handbook of Differential Equations, 3rd ed. Orlando, Florida: Academic Press Inc., 1997.

[9] B. Cochelina and C. Vergez, "A high order purely frequency-based harmonic balance formulation for continuation of periodic solutions," Journal of Sound and Vibration, vol. 324, no. 1-2, pp. 243-262, 2009.

[10] Wang Yongning, Li Heming, Xu Boqiang and Sun Lilhg, "Simulation research of harmonics in electric system of arc furnace," Proc. of the Int. Conf. on Power System Technology POWERCON, 21-24 Nov., Singapore, pp. 902-906, 2004. 